\title{
EL GÉNERO Parabailiella THORAL, 1946 (TRILOBITA) EN EL CÁMBRICO MEDIO DE LAS CADENAS IBÉRICAS (NE DE ESPAÑA)
}

\author{
Juan Bautista CHIRIVELLA MARTORELL ${ }^{1}$, \\ Rodolfo GOZALO ${ }^{l}$ y Eladio LIÑÁN ${ }^{2}$ \\ ${ }^{1}$ Departamento de Geología. Universitat de València. C/ Dr. Moliner, 50. \\ 46100-Burjassot, Valencia.rodolfo.gozalo@uv.es \\ ${ }^{2}$ Departamento Ciencias de la Tierra-IUCA (Paleontología). Facultad de \\ Ciencias. Universidad de Zaragoza. 50009-Zaragoza.
}

Chirivella Martorell, J. B., Gozalo, R. \& Liñán, E. 2009. El género Parabailiella Thoral, 1946 (Trilobita) en el Cámbrico medio de las Cadenas Ibéricas (NE de España). [The genus Parabailiella Thoral, 1946 (Trilobita) from the middle Cambrian of the Cadenas Ibéricas (NE Spain).] Revista Española de Paleontología, 24 (2), 171-184. ISSN 0213-6937.

\begin{abstract}
We analysed the record of the genus Parabailiella Thoral, 1946 in the middle Cambrian of the Cadenas Ibéricas. The four species assigned to the genus have been identified and described in this region for the first time. All the four species are recorded in Jarque 1 section while only some of them are recorded in the rest of the studied sections. The biostratigraphic distribution of the Parabailiella is middle Leonian to basal upper Caesaraugustan, which is equivalent in the International Subcommission on Cambrian Stratigraphy (ISCS) scale to the most part of the Cambrian Stage 5 and to the lower part of the Drumian Stage. The palaeogeographic distribution of the genus is restricted to the Mediterranean region, particularly the European Platform of the Perigondwana region.
\end{abstract}

Key words: Systematic, trilobites, middle Cambrian, biostratigraphy, palaeobiogeography.

\section{RESUMEN}

Se analiza el registro del género Parabailiella Thoral, 1946 en el Cámbrico medio de las Cadenas Ibéricas, al tiempo que se identifican y describen por primera vez en la región las cuatro especies que previamente se habían incluido en este género. La mayoría de las especies se encuentran registradas en varias secciones y las cuatro se han identificado en la sección Jarque 1. La distribución bioestratigráfica del género abarca desde el Leoniense medio hasta la base del Caesaraugustiense superior, lo que equivale en la escala propuesta por la International Subcommission on Cambrian Stratigraphy (ISCS) a la mayor parte del Piso 5 del Cámbrico y a la parte inferior del Piso Drumiense. La distribución paleogeográfica del género es exclusiva de la región Mediterránea, concretamente de la Plataforma Europea de la región perigondwánica.

Palabras clave: Sistemática, trilobites, Cámbrico medio, bioestratigrafía, paleobiogeografía.

\section{INTRODUCCIÓN}

El género Parabailiella fue definido en el Cámbrico medio de la Montaña Negra (Francia) por Thoral (1946) con una sola especie Parabailiella languedocensis Thoral, 1946. Posteriormente fueron descritas por Sdzuy tres nuevas especies en Alemania y en distintas regiones de España (Sdzuy, 1957, 1968); ahora se citan por primera vez las cuatro especies asignadas al género en las $\mathrm{Ca}$ denas Ibéricas, donde presentan una distribución estrati- gráfica más amplia que la previamente conocida en otras regiones.

En este trabajo se revisan las cuatro especies incluidas en Parabailiella, y se asignan algunos taxones previamente determinados en nomenclatura abierta a las mismas. También se discute el nivel jerárquico de Parabailiella, ya que este taxón se ha considerado tanto género como subgénero.

El material estudiado en las Cadenas Ibéricas se ha recolectado en varias secciones, que previamente habían sido analizadas, tanto desde el punto de vista estratigráfi- 
co como bioestratigráfico, por lo que simplemente se van a enumerar e indicar los trabajos donde se describen detalladamente:

- Rambla de Valdemiedes 1 (RV1) y Rambla de Valdemiedes 2 (RV2) de Murero; Liñán \& Gozalo (1986), Gozalo et al. (1996) y Liñán et al. (2008)

- Sección Villafeliche 1 (Vi 1); Gozalo et al. (1993, 1996)

- Área de Ateca, con varias secciones estudiadas por Álvaro Blasco (1994)

- Sección Jarque (J 1); Liñán et al. (1996, 2008) y Chirivella Martorell et al. (2003)
- Área de Borobia, con varias secciones estudiadas por Gámez et al. (1991)

- Sección Mesones 3 (M 3); Valenzuela et al. (1990)

Todas las secciones se localizan en la provincia de $\mathrm{Za}$ ragoza, con la excepción de la de Borobia que se sitúa en la provincia de Soria. Desde un punto de vista geológico todas pertenecen a la Unidad de Badules, excepto la de Mesones, que se enclava en la Unidad homónima (Fig. 1; Gozalo \& Liñán, 1988; Liñán et al., 2008).

Abreviaturas y depósito: En el listado previo de secciones se indica entre paréntesis la sigla de campo de cada una de las

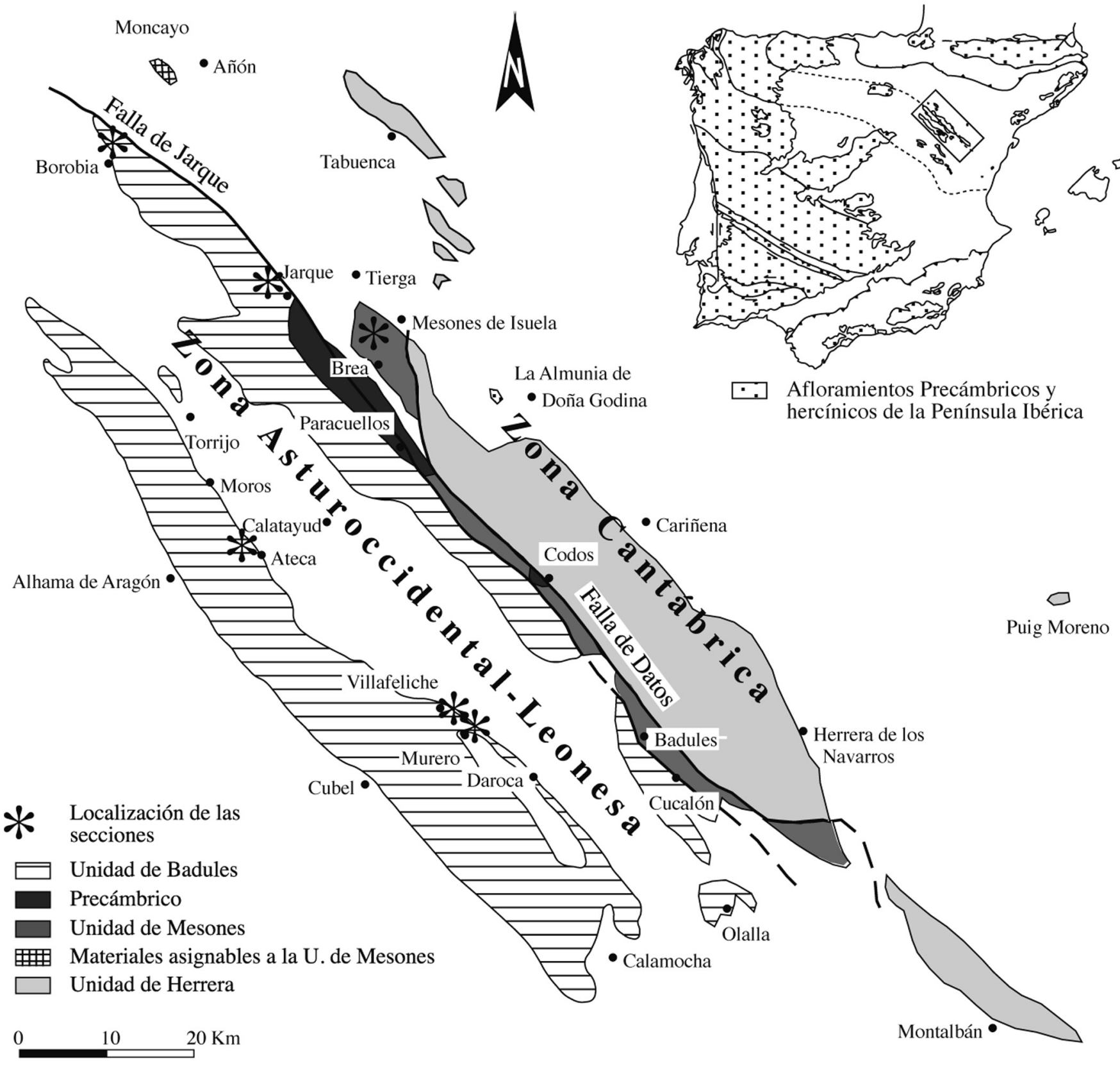

Figura 1. Situación geológica del área de estudio, * situación de las secciones estudiadas (modificado de Gozalo \& Liñán, 1988). Geological setting of the studied area, * location of studied sections (modified from Gozalo \& Liñan, 1988). 
secciones (en las figuras 4 a 7 se incluye esta sigla seguida del número indicativo del nivel del que proceden los ejemplares figurados). El material figurado y/o medido se encuentra depositado en el Museo de Paleontología de la Universidad de Zaragoza (MPZ). Los ejemplares con siglas MPZ3148 a MPZ3168 fueron estudiados y figurados por Liñán \& Gozalo (1986), el nuevo material estudiado en este trabajo está catalogado con las siglas MPZ 2007/1199, 2007/1200, 2007/1216 a 2007/1239 y $2007 / 1241$ a $2007 / 1392$.

\section{ESTRATIGRAFÍA Y BIOESTRATIGRAFÍA}

Todo el material de Parabailiella estudiado en las Cadenas Ibéricas se ha recolectado en la parte alta de la Formación Mansilla y en la mitad inferior de la Formación Murero, ambas del Grupo Mesones (Liñán et al., 1992, 2002, 2004, 2008). En la figura 2 se ofrece un esquema de la distribución bioestratigráfica de las cuatro especies en relación con la zonación establecida para la región Mediterránea (Sdzuy, 1972; Liñán \& Gozalo, 1986; Sdzuy et al., 1999; Liñán et al., 2002, 2008; Esteve et al., 2008) y su comparación con las nuevas propuestas de la International Subcommission on Cambrian Stratigraphy (ISCS; Babcock et al., 2007), que correspondería a parte de los pisos 5 y Drumiense (Gozalo et al., 2008, 2009).

Tres de las cuatro especies de Parabailiella aparecen simultáneamente (Fig. 2) en las Cadenas Ibéricas ( $P$. schmidti, P. matutina y P. sebarensis). Los niveles previos a este registro, se corresponden a los materiales depositados durante la regresión del Leoniense medio (Liñán et al., 1997, 2002), que no han librado trilobites. De este modo, el primer nivel donde se registran estas especies, junto con otra diversa fauna de trilobites, braquiópodos, etc, realmente marca la colonización del área por faunas procedentes de regiones cercanas, como por ejemplo la Cordillera Cantábrica. Este nivel corresponde a la zona de Eccaparadoxides asturianus en el Leoniense superior (ver Sdzuy et al., 1999). La aparición de las especies P. matutina y P. sebarensis en niveles más antiguos en la Cordillera Cantábrica (Sdzuy, 1968) y la Sierra de Córdoba (zona de Eccaparadoxides sdzuyi, ver Liñán et al., 1995) hablan a favor de esta colonización. Desde un punto de vista paleogeográfico, las Cadenas Ibéricas conformaban un golfo cuya conexión con mar abierto era la región Cantábrica, por lo que la colonización por parte de los trilobites, cuando las condiciones ambientales fueron las adecuadas, se produciría desde esta región.

Las especies $P$. matutina y $P$. sebarensis se extinguen simultáneamente en la zona de Badulesia tenera en el Caesaraugustiense inferior (Fig. 2); para ambas especies supone aumentar su rango de distribución conocido, ya que hasta ahora se consideraban exclusivas del Leoniense, y probablemente quedaron un tiempo como especies relictas en la Cordillera Ibérica antes de su desaparición.
La especie $P$. schmidti presenta una distribución que se extendería probablemente desde la zona de Eccaparadoxides sdzuyi hasta la base de Pardailhania hispida,

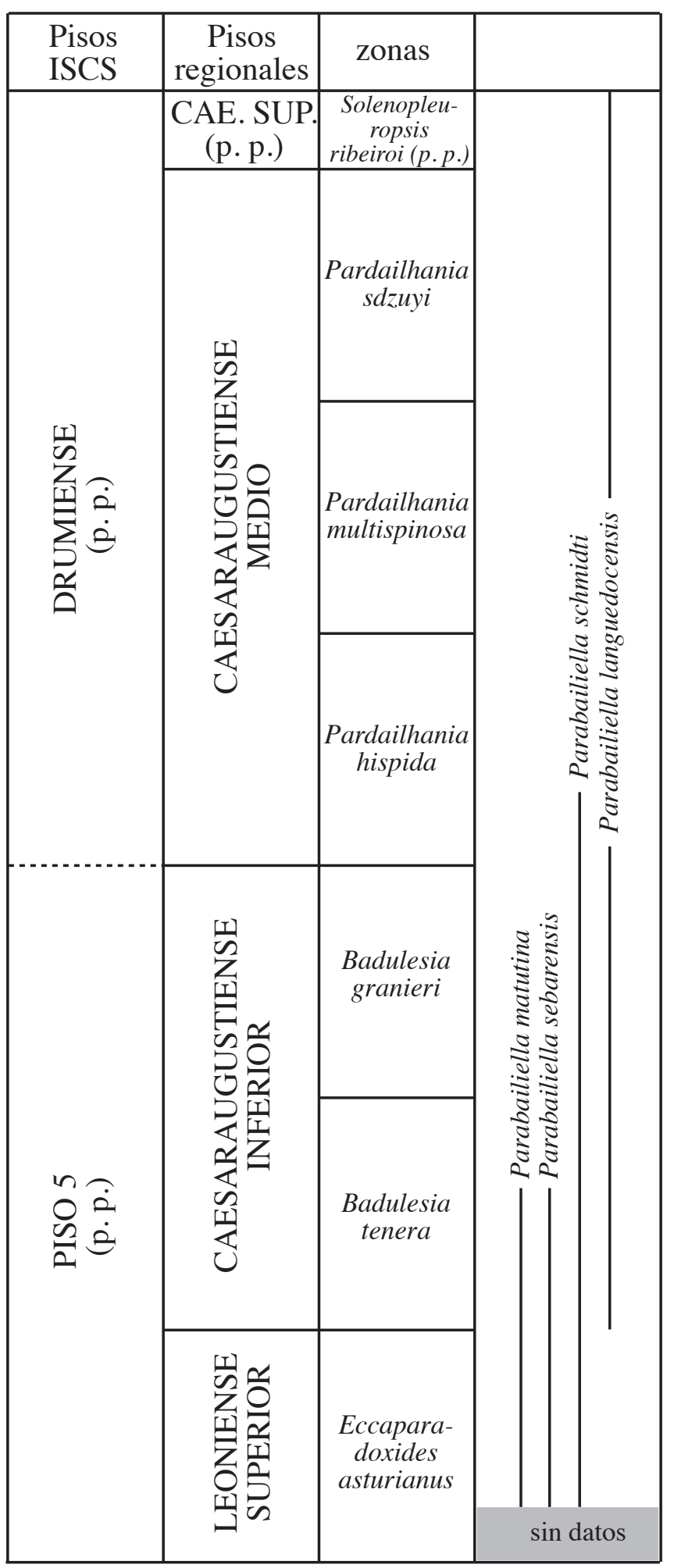

Figura 2. Distribución estratigráfica de las especies de Parabailiella Thoral, 1946 en las Cadenas Ibéricas. Stratigraphical distribution of the Parabailiella Thoral, 1946 species in the Cadenas Ibéricas. 
esto es desde el Leoniense medio hasta la base del Caesaraugustiense medio (Fig. 2). El rango de distribución de esta especie también se ve aumentado tanto por los nuevos datos de las Cadenas Ibéricas como por la inclusión del taxón de Chequia figurado como Conocoryphe aff. languedocensis por Valíček \& Szabad (2002) ya que hasta ahora sólo se había encontrado en las zonas de Eccaparadoxides asturianus y Badulesia tenera (ver Sdzuy, 1957, 1968, 1972).

Por último, la especie P. languedocensis presenta un rango que va desde la zona de Badulesia tenera hasta la base de la zona de Solenopleuropsis ribeiroi (ver Sdzuy, 1972; Liñán \& Gozalo, 1986).

\section{SISTEMÁTICA}

Orden PTYCHOPARIIDA Swinnerton, 1915

Suborden PTYCHOPARIINA Richter, 1932

Superfamalia Ptychoparioidea Matthew, 1888

\section{Famila Conocoryphidae Angelin, 1854}

Comentarios: Cotton (2001) realizó una revisión y análisis filogenético de un conjunto de pticopáridos ciegos, entre los que se incluían los conocorífidos. La familia Conocoryphidae, en el sentido de Cotton (op. cit.) está ampliamente representada en los materiales del Cámbrico medio de la provincia Acadobáltica (sensu Sdzuy, 1972) y en Siberia (ver Korobov, 1973), e incluiría: Conocoryphe Hawle \& Corda, 1847 (=Couloumania Thoral, 1946); Ctenocephalus (Ctenocephalus) Hawle \& Corda; 1847; Elyx Angelin, 1854; Bailiella Matthew, 1885; Ctenocephalus (Hartella) Mathew, 1885; Cainatops Matthew, 1899 (=Cornucoryphe Sdzuy \& Liñán, 1996); Bailiaspis (Bailiaspis) Resser, 1936; Parabailiella Thoral, 1946; Bailiaspis (Tchaispis) Korobov, 1966; y por último, se añadió a la familia el género Catharia Álvaro \& Vizcaïno, 2003 [fue posteriormente denominado por Álvaro (2007) como Occatharia por homonimia con Catharia Lederer, 1863, un lepidóptero; Özdikmen (2009) propone el nombre Novocatharia, por los mismos motivos indicados por Álvaro (2007), pero en este caso tiene prioridad el nombre propuesto por este último autor].

Respecto a los taxones incluidos en Conocoryphidae por Cotton (2001) todos los autores están de acuerdo en esa inclusión, aunque existen discrepancias en algunos de los taxones que este autor excluye de la familia como es el caso del género Holocephalina Salter, 1864, que Kim et al. (2002) sí incluyen en la familia, inclusión con la que estamos de acuerdo.

Dentro de los conocorífidos hay tres géneros: Conocoryphe, Bailiella y Parabailiella que presentan características muy similares, sobre todo entre los dos primeros; el análisis de Cotton (2001) muestra escasa evidencia de la monofilia de las especies analizadas de estos dos géneros, y existen problemas para diferenciarlos por sus caracteres morfológicos.

La distinción entre los tres géneros citados se realiza, principalmente, en función de dos caracteres (Westergård, 1950; Courtessole, 1973; Jell \& Hughes, 1997; Cotton, 2001): 1) la posición de la sutura facial afectando al surco marginal (Bailiella y Parabailiella) o sólo afectando al borde marginal (Conocoryphe) y 2) la presencia de dos surcos preglabelares divergentes que delimitan el área preglabelar del resto de la fixígena ausente en Bailiella y que puede estar presente o no en Conocoryphe y Parabailiella. Para la mayoría de los autores, incluido Cotton (2001), el primer carácter es considerado como el más importante y, posiblemente, de nivel genérico. En el caso de Bailiella, el análisis filogenético de Cotton (2001) muestra que este género es claramente polifilético, al menos tal y como se usa en la actualidad, en el que por ejemplo se incluye Bailiella lantenoisi (Mansuy, 1916) cuya sutura facial no afecta al surco marginal (ver Jell \& Hughes, 1997). Kim et al. (2002) comentan que el género Bailiella no presenta caracteres diagnósticos claros, por lo que lo utilizan con reservas a la espera de una futura revisión del mismo.

\section{Género Parabailiella Thoral, 1946}

Especie tipo: Parabailiella languedocensis Thoral, 1946

Comentarios: La posición de la sutura es el carácter que permite distinguir claramente los géneros Conocoryphe y Parabailiella. Pero muchas veces la conservación fragmentaria de los cranidios impide observar este carácter, por este motivo Sdzuy (1957) propuso considerar Parabailiella como un subgénero de Conocoryphe, por una cuestión práctica, ya que así se facilitaba, al menos, la asignación genérica. Este criterio utilitario ha sido aceptado por numerosos autores posteriormente, como por ejemplo: Sdzuy (1961, 1968), Courtessole (1973), Shergold \& Sdzuy (1984), Liñán \& Gozalo (1986), etc. De todos modos, los últimos análisis de la familia parecen evidenciar que se trata de un carácter suficientemente importante para considerarlo como de rango genérico, y en este sentido $\mathrm{Pa}$ rabailiella es considerado como género en este trabajo de acuerdo con la definición original de Thoral (1946) y el uso de Cotton (2001) y Álvaro \& Vizcaïno (2003).

Distribución geográfica y paleogeográfica: Además del registro en las Cadenas Ibéricas que se analiza en este trabajo con nuevos datos, el género Parabailiella también se ha encontrado en la Montaña Negra (Francia), Doberlug (Alemania), Cordillera Cantábrica, Sierra de Córdoba y Sierra de la Demanda (España), Montañas de Amanos y Sultán (Turquía), Bohemia (República Checa) y Anti Atlas (Marruecos). Su distribución geográfica está limitada a la subprovincia Mediterránea (Sdzuy, 1972; Sdzuy et al., 
1999). En la figura 3 se muestran los hallazgos de Parabailiella representados sobre la reconstrucción paleogeográfica de Courjault-Rade et al. (1992), donde se puede constatar que todos los registros del género se sitúan en la Plataforma Europea de la región perigondwánica, en latitudes tropicales y subtropicales.

Aspectos sinecológicos: El único análisis cuantitativo y estadístico estrato por estrato y en el que se reflejaron los porcentajes relativos de los grupos de trilobites dominantes (entre ellos la relación existente entre Paradoxídidos y Conocorífidos) ha sido el realizado en las secciones de Murero por Liñán \& Gozalo (1986) y en las secciones aquí referenciadas en Chirivella Martorell (2008). De los datos de estos dos trabajos es fácil deducir que desde el Leoniense superior hasta la base del Caesaraugustiense superior es posible reconocer en las Cadenas Ibéricas dos comunidades que se van relevando en el tiempo: la comunidad de Eccaparadoxides y la comunidad de Parabailiella. La primera vez que Parabailiella desplaza como género dominante de la comunidad a Eccaparadoxides acontece durante la zona de Badulesia tenera y gran parte de la zona de Badulesia granieri. La segunda vez que domina la

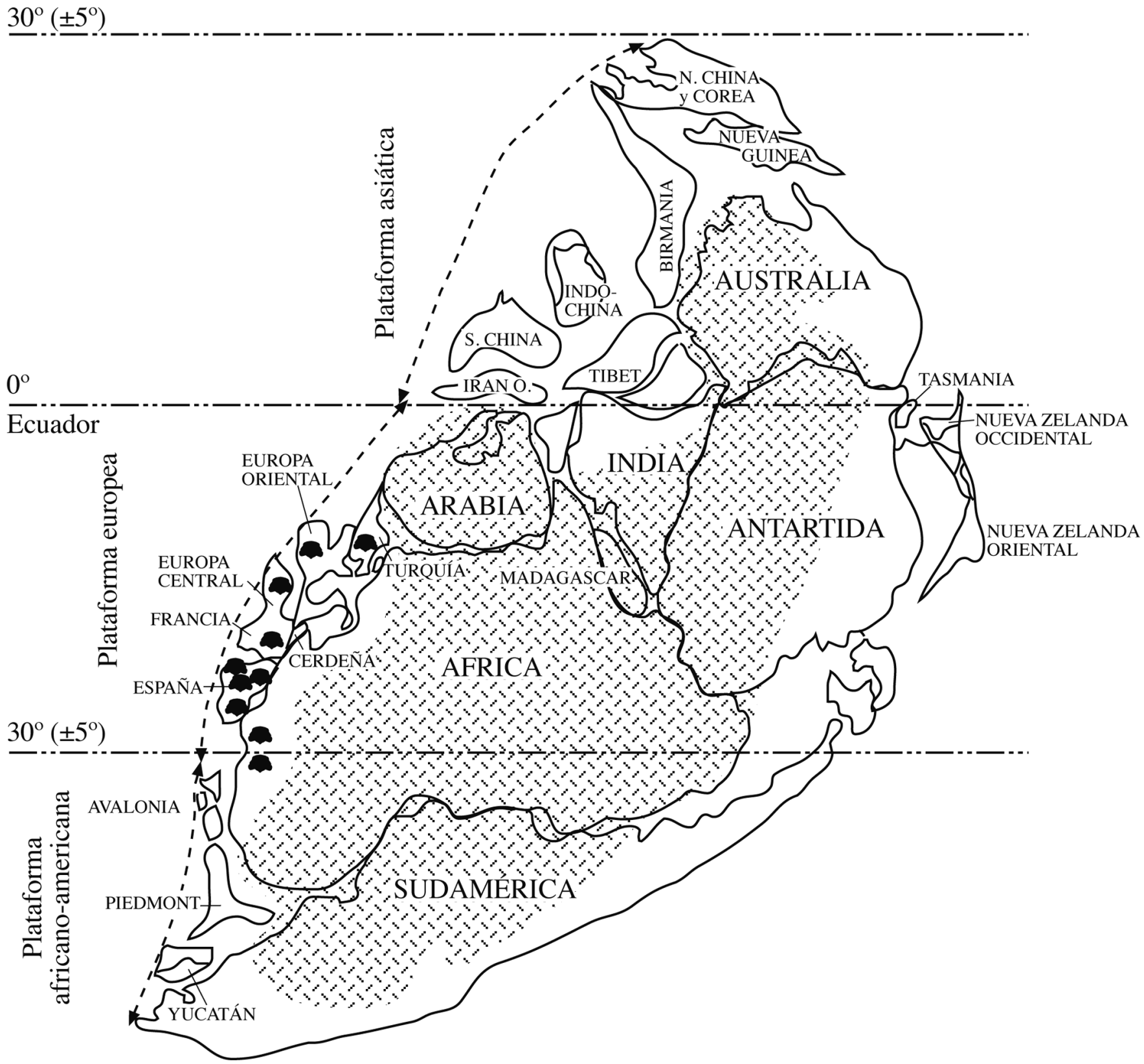

Figura 3. Distribución paleogeográfica de las especies de Parabailiella Thoral, 1946, dibujadas sobre la reconstrucción de CourjaultRadé et al. (1992).

Palaeogeographic distribution of Parabailiella Thoral, 1946 species plotted on the reconstruction of Courjault-Radé et al. (1992). 
comunidad de Parabailiella en el ecosistema del Cámbrico medio de Murero ocurre en la parte alta de la zona de Pardailhania hispida (Fig. 2). Una tercera vez aconteció en la parte media de la zona de Pardailhania multispino$s a$ (según se ha podido constatar en las secciones RV1 y RV2). Las causas ecológicas de estos sucesivos cambios en la estructura de la comunidad del Cámbrico medio de Murero, donde los trilobites eran el grupo mayoritario es un tema a desarrollar en el futuro.

Distribución estratigráfica: La distribución bioestratigráfica del subgénero Parabailiella en la subprovincia Mediterránea comprende desde la zona de Eccaparadoxides sdzuyi (Leoniense medio) hasta la base de la zona de Solenopleuropsis ribeiroi (base del Caesaraugustiense superior), esto es el Piso 5 del Cámbrico y base del Drumiense en la escala propuesta por la ISCS.

Además de estas cuatro especies, Dean et al. (1986) citan Conocoryphe (Parabailiella) sp. asociada a Badulesia tenera en Turquía. Geyer et al. (1995) y Geyer \& Landing (2006) citan Conocoryphe (Parabailiella) sp. en las zonas de Ornamentaspis frequens y Kimataspis arenosa en el Antiatlas marroquí. Estos mismos autores, señalan la presencia de Conocoryphe (Parabailiella) sp. aff. Conocoryphe (Parabailiella) schmidti en la zona de Badulesia tenera de la misma región. La distribución de estos taxones es coherente con la distribución general encontrada para el género en las otras regiones, ya que abarcaría desde el Leoniense medio hasta el Caesaraugustiense inferior (para una correlación ver Sdzuy et al., 1999; Geyer \& Landing, 2004; Gozalo et al., 2007).

\section{Parabailiella languedocensis Thoral, 1946 Figura 4}

v 1986. Conocoryphe (Parabailiella) languedocensis Thoral, 1946.- Liñán \& Gozalo, 75, lám. 29 figs. 6-14, lám. 30 figs. 1-11, lám. 31 fig. 1 (con sinónimos anteriores).

v 1991. Conocoryphe (Parabailiella) languedocensis Thoral, 1946.- Gámez, Fernández-Nieto, Gozalo, Liñán, Mandado \& Palacios, lám. I fig. 3.

1999. Conocoryphe (Parabailiella) languedocensis.- Álvaro, Vizcaïno \& Vennin, fig. 5 C.

2000. Conocoryphe (Parabailiella) languedocensis.- Álvaro \& Vizcaïno, fig. 1.

2001. Parabailiella languedocensis Thoral, 1946.- Cotton, lám. 4 fig. 1.

v 2001. Conocoryphe (Parabailiella) languedocensis Thoral, 1946.- Liñán \& Sdzuy, 260-261, figs. 7.e, 7.f.

Material: 2 ejemplares casi completos y unos 130 cranidios conservados como moldes internos y externos en margas dolomíticas de variados colores: verdes, rojizas y ocres claros, en lutitas ocres, rojas y verdes con algunos nódulos carbonatados dispersos y dolomias ocres. Los ejemplares estudiados en este trabajo tienen los siguientes números de registro MPZ 3148 al 3168, MPZ 1321 y MPZ 2007/1241 al 2007/1334.
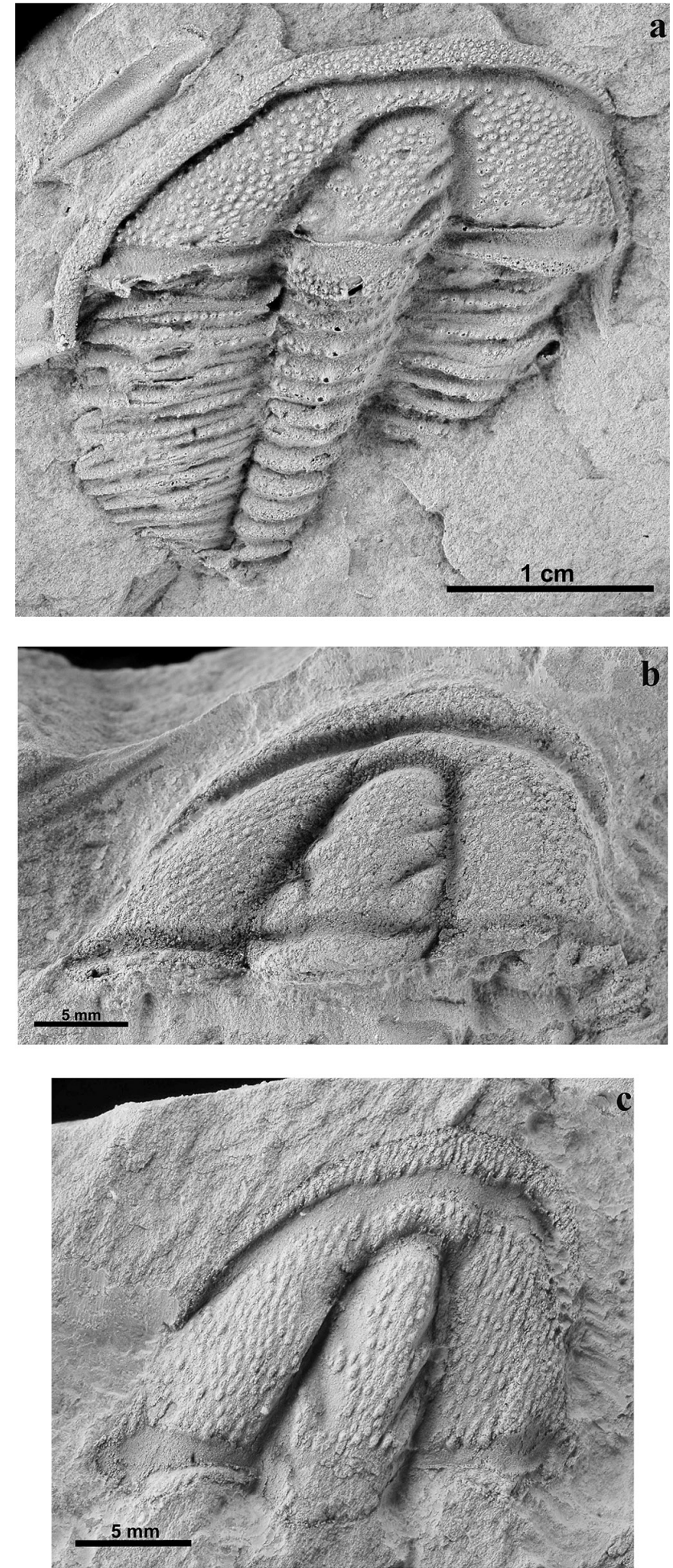

Figura 4. Parabailiella languedocensis Thoral, 1946. a, réplica en látex de un ejemplar casi completo, MPZ2007/1321, RV2/13.2. b, molde interno de un cranidio, MPZ2007/1285, RV1/13.1. c, molde interno de un cranidio, MPZ2007/1282, RV1/13.1.

Parabailiella languedocensis Thoral, 1946. a, latex cast of an almost complete, MPZ2007/1321, $R V 2 / 13.2$. b, internal mould of a cranidium, MPZ2007/1285, RV1/13.1.c internal mould of a cranidium, MPZ2007/1282, RV1/13.1. 
Descripción: Cefalón semicircular, margen anterior arqueado, margen posterior recto con los extremos genales ligeramente arqueados hacia atrás.

Cranidio semicircular con sutura marginal. El borde es arqueado, abombado y ligeramente apuntado en su parte central, es inhomogéneo siendo más ancho en el centro y disminuyendo hacia los extremos. Presenta ornamentación consistente en tubérculos desordenados, más finos que en el resto del cranidio, dispuestos en tres o cuatro filas en la parte central del borde que disminuyen hacia los laterales hasta quedar una sola fila. El surco anterior es liso, ancho y profundo, es casi homogéneo en todo su recorrido estrechándose ligeramente en los extremos y se interrumpe al llegar a la sutura facial, la cual no corta al surco posterior. El área preglabelar es abombada y está recorrida por dos o tres filas de tubérculos gruesos desordenados, presenta dos constricciones en sus laterales a la altura de los extremos del ápice de la glabela, en las que continúa la ornamentación; son más o menos profundas según ejemplares pero no llegan a separar el área preglabelar de las fixígenas. La glabela es cónica con el ápice truncado y redondeado, está recorrida por tres pares de surcos no transglabelares que se dirigen hacia atrás siendo el posterior el de mayor longitud, presenta ornamentación consistente en tubérculos grandes y desordenados. Anillo occipital en forma de semicírculo invertido, separado del resto de la glabela por un surco occipital profundo y amplio con forma convexa hacia la parte anterior; el anillo occipital presenta la misma ornamentación que el resto del cranidio; en la parte central posterior del anillo occipital se observa, en algunos ejemplares, un tubérculo más grueso que los demás, a modo de espina occipital residual. Fixígenas abombadas que presentan la misma ornamentación en tubérculos que el resto del cranidio, no se observan aristas oculares; el surco posterior es liso, tiene forma ovalada que se ensancha al llegar cerca del ángulo genal y en su parte posterior presentan una expansión dirigida hacia atrás.

Librígenas estrechas, casi sin área lateral, que terminan en una corta y robusta espina genal que generalmente no sobrepasa el cuarto segmento torácico.

El tórax más completo presenta 14 segmentos, la anchura del raquis es aproximadamente la mitad de una pleura y está fuertemente abombado, los surcos pleurales son anchos y están flanqueados por una fila de tubérculos, los extremos de las pleuras son redondeados, con la parte anterior biselada y la posterior recta.

Pigidio elipsoidal; el raquis presenta tres surcos flanqueados cada uno de ellos por una fila de tubérculos (cuatro en total), los surcos y las filas de tubérculos se extienden por el campo pleural, los tubérculos se encuentran sobre una elevación en forma de cresta que no supera en altura al raquis. Los surcos laterales del raquis son profundos y anchos. El borde del pigidio es redondeado y no presenta ninguna expansión.

Observaciones: Los ejemplares aquí estudiados se encuentran dentro de la variabilidad intraespecífica asignada para esta especie en los trabajos de Sdzuy (1961), Courtessole (1973) y Liñán \& Gozalo (1986). En estos trabajos se acepta una variabilidad importante, sobre todo en la posición de la sutura, la cual siempre afecta al surco lateral pero que en ocasiones corta al surco posterior (Fig. 4.c) y otras presenta un posición más anterior y el surco posterior se encuentra completo, observándose como se cierra en la región posterolateral del cranidio (ver Liñán \& Gozalo, 1986: lám. 31 figs. 7-9). La variabilidad morfológica se hace más amplia en los niveles estratigráficos más altos, como ya comentan Liñán \& Gozalo (1986). Esta variabilidad se observa en ejemplares procedentes de los mismos niveles estratigráficos, por lo que se considera como variación intraespecífica.

Distribución estratigráfica: En las Cadenas Ibéricas esta especie se ha encontrado en: nivel 3 de la Sección Ateca 2; niveles 5 y 6 de la Sección Mesones 3; niveles 6, 8 y 9 de la Sección Villafeliche 1; niveles 7 y 8 de la Sección Borobia 2; niveles 5 y 8 de la Sección Borobia 3; Niveles 156 al 196 de la Sección Jarque 1; niveles 11D al 15 de la sección RV1, y niveles 13.1 y 13.2 de la sección RV2. De acuerdo con esta distribución estratigráfica el taxón se registra en las Cadenas Ibéricas desde la zona de Badulesia tenera hasta la base de la zona de Solenopleuropsis ribeiroi (ver Liñán \& Gozalo, 1986; Liñán et al., 2008).

Esta especie fue definida en la Montaña Negra, donde se registra en los niveles A1 y A2 con Pardailhania hispida y P. multispinosa, de acuerdo con Courtessole (1973). En las montañas del Sultán (Sultan Dă̆; Turquía central) Shergold \& Sdzuy (1984) la citan en la fauna de Pardailhania junto con la especie $P$. hispida, entre otras.

La distribución estratigráfica de esta especie abarcaría todo el Caesaraugustiense inferior, medio y la base del superior; correlacionable con la parte alta del Piso 5 del Cámbrico y la parte baja del Drumiense.

\section{Parabailiella matutina Sdzuy, 1968} Figura 5

\section{* 1968. Conocoryphe (Parabailiella) matutina n. sp.- Sdzuy, 126-128, lám. 9 fig. 15, lám. 10 figs. 1-17, 19. \\ p 1978. Conocoryphe (Parabailiella) aff. matutina Sdzuy, 1968.- Liñán Guijarro, 191-192, lám. XII figs. 2-3.}

Material: un cefalón y 36 cranidios conservados como moldes internos y externos en margas dolomíticas de color variado: verdes, rojizas y ocres claros, en lutitas ocres, rojas y verdes con algunos nódulos carbonatados dispersos y dolomías ocres. Los ejemplares estudiados tienen número de registro MPZ 2007/1365 al $2007 / 1392$.

Descripción: Cranidio semicircular con sutura marginal, con el margen anterior arqueado y el posterior recto. El borde es arqueado y ligeramente apuntado en su parte central, es inhomogéneo siendo más ancho en el centro y disminuyendo hacia los extremos, presenta ornamentación consistente en tubérculos dispuestos en dos o tres filas en la parte central que disminuyen hacia los laterales hasta quedar una sola fila. El surco anterior es más ancho que profundo y no presenta ornamentación. El surco preglabelar es más profundo que ancho y delimita claramente a la glabela en todo su contorno, no presenta ornamentación. El área preglabelar está ligeramente abombada no superando en altura a las fixígenas ni a la glabela, está recorrida por 
dos filas de tubérculos finos y desordenados que se extienden por las fixígenas. La glabela es cónica con el ápice redondeado, está recorrida por tres pares de surcos no transglabelares que se dirigen hacia atrás y muy ligeramente marcados, presenta ornamentación consistente en tubérculos desordenados. Anillo occipital en forma de semicírculo invertido, recorrido con la misma ornamentación que el resto del cranidio, presenta un tubérculo occipital más engrosado en su parte central posterior. Fixígenas
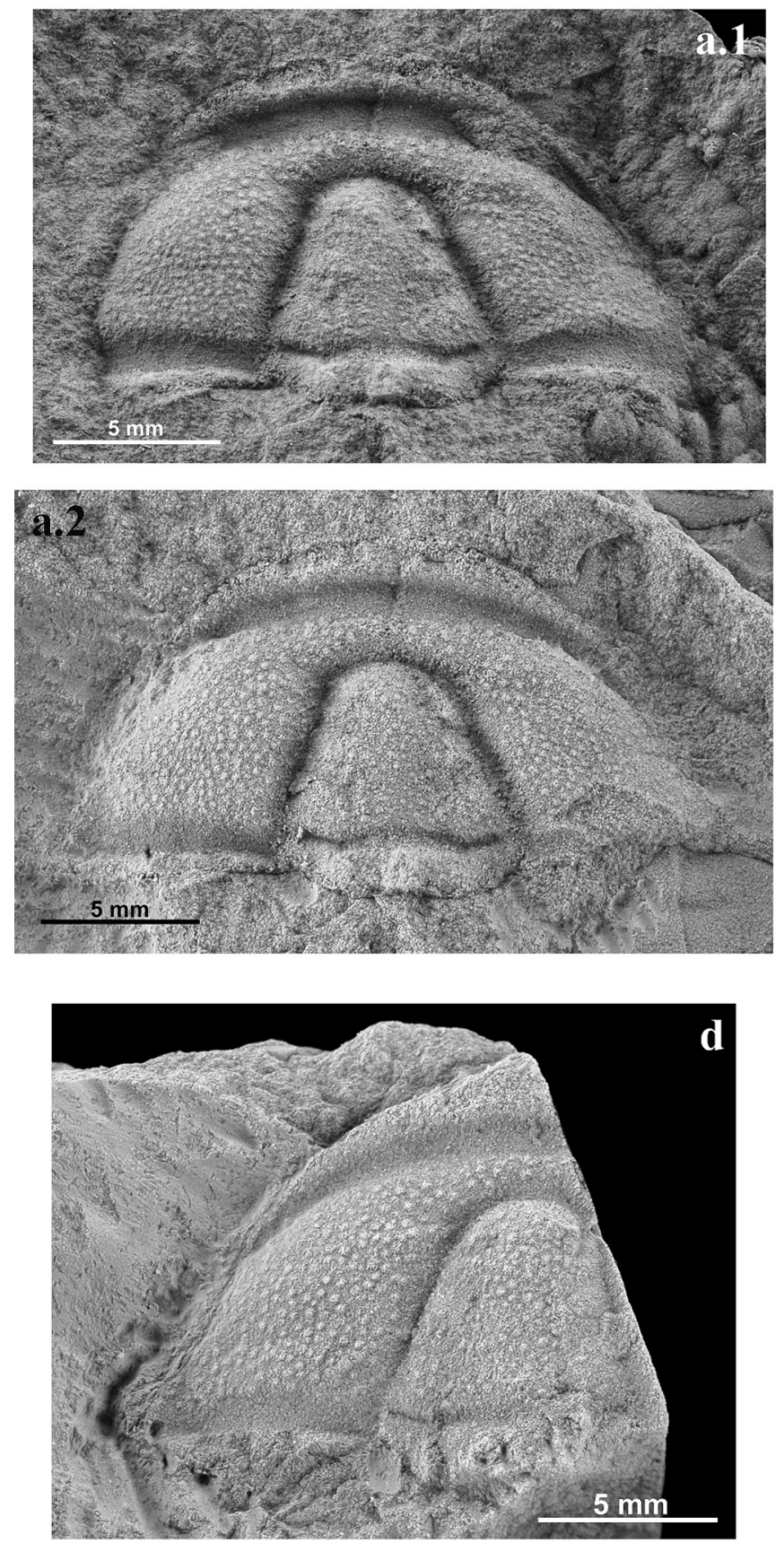

abombadas sin superar en altura a la glabela, presentan la misma ornamentación en tubérculos que el resto del cranidio, junto al surco lateral de la glabela a una altura ligeramente inferior al ápice de la glabela, presenta una corta arista ocular rudimentaria muy débil. El surco posterior es recto, ancho y liso.

Observaciones: Los ejemplares asignados a $P$. matutina en este trabajo presentan las características diagnósticas
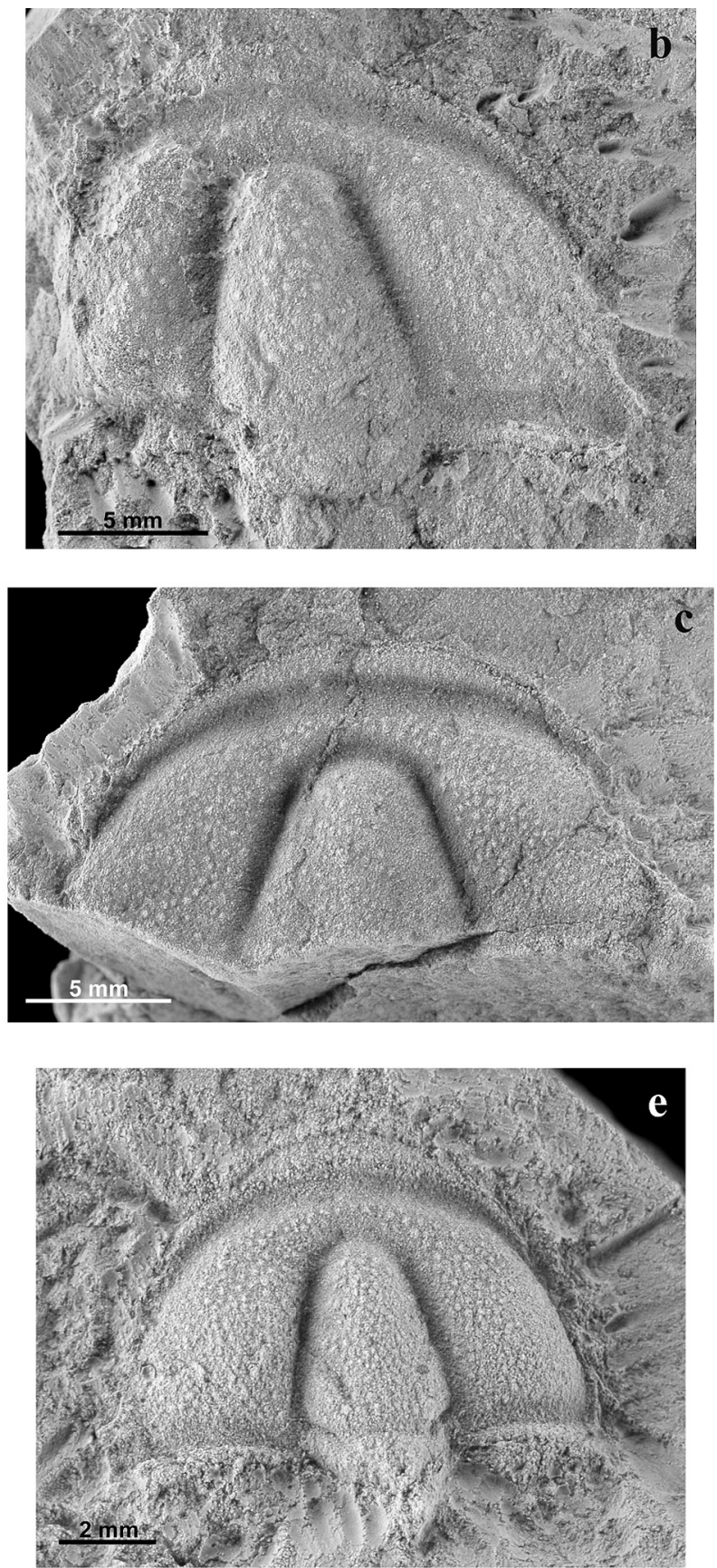

Figura 5. Parabailiella matutina Sdzuy, 1968. a, cranidio, MPZ 2007/1374, J1/147F; a.1, réplica de látex; a.2, molde interno. b, molde interno de un cranidio, MPZ2007/1371, Vi1/2. c, molde interno de un cranidio, MPZ2007/1376, J1/147F. d, molde interno de un fragmento de cranidio, MPZ2007/1378, J1/147F. e, molde interno de un cranidio, MPZ2007/1380, J1/147F. Parabailiella matutina Sdzuy, 1968. a, cranidium, MPZ 2007/1374, J1/147F; a.1, latex cast; a.2, internal mould. b, internal mould of a cranidium, MPZ2007/1371, Vi1/2 . c, internal mould of a cranidium, MPZ2007/1376, J1/147F. d, internal mould of a cranidium fragment, MPZ2007/1378, J1/147F. e, internal mould of a cranidium, MPZ2007/1380, J1/147F. 
de la especie: poseer unas aristas oculares rudimentarias y presentar dos filas de tubérculos en la parte central del área preglabelar (ver Sdzuy, 1968).

Liñán Guijarro (1978) y Liñán et al. (1995) encontraron unos pocos cranidios de Parabailiella en varios niveles de la sección Fuente Bernardo (Sierra de Córdoba); todos ellos presentan una arista ocular rudimentaria pero observaron una variación importante en la anchura del área preglabelar (sag.) y en el número de filas de tubérculos en la misma, por lo que optaron por clasificarlos como Conocoryphe $(P$.) aff. matutina. En esta revisión se ha decido utilizar las especies tal y como fueron caracterizadas por Sdzuy (1968) en la Cordillera Cantábrica. Este autor las diferenció básicamente por el número de filas de tubérculos en el área preglabelar y por que las especies no se encontraban juntas en los yacimientos por él estudiados; sin embargo, tanto en las Cadenas Ibéricas como en la Sierra de Córdoba estas dos especies se registran juntas en varios niveles. Un pigidio aislado muestra el perfil del margen posterior que forma un ligero ángulo obtuso y cuyo vértice estaría a la altura del eje sagital del raquis (Liñán Guijarro, 1978, lám. XII, fig. 6).

Distribución estratigráfica: En las Cadenas Ibéricas esta especie se ha encontrado en el nivel 45 de la Sección Borobia 1, nivel 6 de la Sección Borobia 2, nivel 5 de la Sección Borobia 3, niveles 1 al 4 de la Sección Villafeliche 1, niveles $147 \mathrm{~F}$ al 164 de la Sección Jarque 1, nivel 1.2 la sección Mesones 3, nivel 5B de la Sección Ateca 1 y niveles 10-11 de la Sección Ateca 4. Su distribución para esta región iría desde la zona de Eccaparadoxides asturianus hasta la base de Badulesia tenera.

La distribución de esta especie en la Cordillerra Cantábrica y en la Sierra de Córdoba se inicia en la zona de $E c$ caparadoxides sdzuyi y llega hasta la de E. asturianus (Sdzuy, 1968; Liñán et al., 1995; Perejón et al., 1996; Gozalo et al., 2003). En conjunto tendría una distribución que iría desde el Leoniense medio hasta la base del Caesaraugustiense, equivalente a la mayor parte del Piso 5 del Cámbrico.

\section{Parabailiella schmidti Sdzuy, 1957} Figura 6

1942. Bailiella heberti (Mun.-Chalm. \& Berg.).- Schmidt, 364-366, lám. 23 figs. 20-23, lám. 24 figs. 1-5.

* 1957. Conocoryphe (Parabailiella) schmidti n. sp.- Sdzuy, 23-24, fig. 13.

1968. Conocoryphe (Parabailiella) cf. schmidti Sdzuy, 1957.- Sdzuy, 129, lám. 10 fig. 18.

2002. Parabailiella cf. languedocensis Thoral, 1946.Valíček \& Szabad, 79-80, lám. 1 figs. 3-4.

Material: Un ejemplar completo, 35 cranidios conservados como moldes internos y externos en margas dolomíticas de color variados: verdes, rojizas y ocres claros, en lutitas ocres, rojas y verdes con algunos nódulos carbonatados dispersos y dolomías ocres. Los ejemplares estudiados tienen número de registro MPZ 2007/1199 y 2007/1200, y MPZ 2007/1216 al 2007/1239.
Descripción: Cefalón semicircular, margen anterior arqueado, margen posterior recto y una corta espina genal.

Cranidio semicircular con sutura marginal que generalmente no corta al surco posterior. El borde es arqueado, abombado y ligeramente apuntado en su parte central, es inhomogéneo siendo más ancho en el centro y disminuyendo hacia los extremos; presenta ornamentación consistente en tubérculos desordenados, más finos que en el resto del cranidio, dispuestos en tres o cuatro filas en la parte central del borde que disminuyen hacia los laterales hasta quedar una sola fila. El surco anterior es liso, ancho y profundo, es casi homogéneo en todo su recorrido estrechándose ligeramente en los extremos, no corta al surco posterior. El área preglabelar es abombada y está recorrida por dos o tres filas de tubérculos gruesos desordenados; presenta dos surcos preglabelares divergentes que se inician a la altura de los extremos del ápice de la glabela, en las que continúa la ornamentación; son más o menos profundas según ejemplares pero no llegan a separar el área preglabelar de las fixígenas. La glabela es cónica con el ápice truncado y redondeado, está recorrida por tres pares de surcos no transglabelares que se dirigen hacia delante siendo el posterior el de mayor longitud; presenta ornamentación consistente en tubérculos grandes y desordenados. Anillo occipital en forma de semicírculo invertido, recorrido con la misma ornamentación que el resto del cranidio, en la parte central posterior del anillo occipital se observa, en algunos ejemplares, el tubérculo occipital, que es más grueso que los demás. Fixígenas abombadas que presentan la misma ornamentación en tubérculos que el resto del cranidio, se observan aristas oculares bien desarrolladas, que en su parte posterior presentan una expansión dirigida hacia atrás hasta llegar a la altura del primer surco glabelar, e incluso el segundo (Fig. 6.e).

Librígenas estrechas, planas y sin ornamentación, terminan en una corta y robusta espina genal que generalmente no sobrepasa el cuarto segmento torácico.

El tórax más completo encontrado presenta 14 segmentos. La anchura del raquis es aproximadamente la mitad de una pleura, los surcos pleurales son anchos y están flanqueados por una fila de tubérculos, los extremos de las pleuras son redondeados.

Pigidio elipsoidal; el raquis presenta tres surcos flanqueados cada uno de ellos por una fila de tubérculos (cuatro en total), los surcos y las filas de tubérculos se extienden por el campo pleural, los tubérculos se encuentran sobre una elevación en forma de cresta que no supera en altura al raquis. Los surcos laterales del raquis son profundos y anchos. El borde del pigidio es redondeado y no presenta ninguna expansión.

Observaciones: Como indica Sdzuy (1957), la morfología de esta especie es muy parecida a $P$. languedocensis, pero la presencia de aristas oculares rudimentarias las separa claramente. Respecto a las especies $P$. matutina y $P$. sebarensis, la principal diferencia es la presencia de los surcos preglabelares divergentes, que no están presentes en estas dos especies definidas en la Cordillera Cantábrica. El nuevo material de las Cadenas Ibéricas presenta una cierta variabilidad morfológica en la anchura del área preglabelar, y en la densidad de la ornamentación, que aquí se consideran de tipo intraespecífico, y dentro de ella se encuentra tanto el material tipo de Doberlug (Alemania) como el ejemplar figurado como $P$. cf. schmidti por Sdzuy (1968) en la Cordillera Cantábrica. 
Valíček \& Szabad (2002) citan Parabailiella cf. languedocensis en la localidad de Jince-Vinice (República Checa). En los dos ejemplares completos que figuran se observa la presencia de aristas oculares rudimentarias, lo que junto a la presencia de surcos preglabelares divergentes claramente marcados permite incluir estos ejemplares dentro de la especie de P. schmidti.

Distribución estratigráfica: En las Cadenas Ibéricas esta especie se ha encontrado en los niveles 1.2 y 2 de la Sección Mesones 3, niveles 5 y 8 de la Sección Borobia 3, niveles
147F al 159 de la Sección Jarque 1, nivel 11 de la Sección Ateca 4, y niveles 11D y 13.1 del corte RV1. Su distribución para esta región iría de la zona de Eccaparadoxides asturianus hasta la base de la zona de Pardailhania hispida.

La especie nominal se encuentra en Doberlug (Alemania) en la zona de Badulesia tenera (ver Sdzuy, 1957) y en la Cordillera Cantábrica con Acadolenus en niveles por debajo de la zona de Eccaparadoxides asturianus (ver Sdzuy, 1968).

Recientemente, Valíček \& Szabad (2002) citan Parabailiella cf. languedocensis en el área de Jince en la República Checa, en el horizonte de Ornamentaspis - Cono-
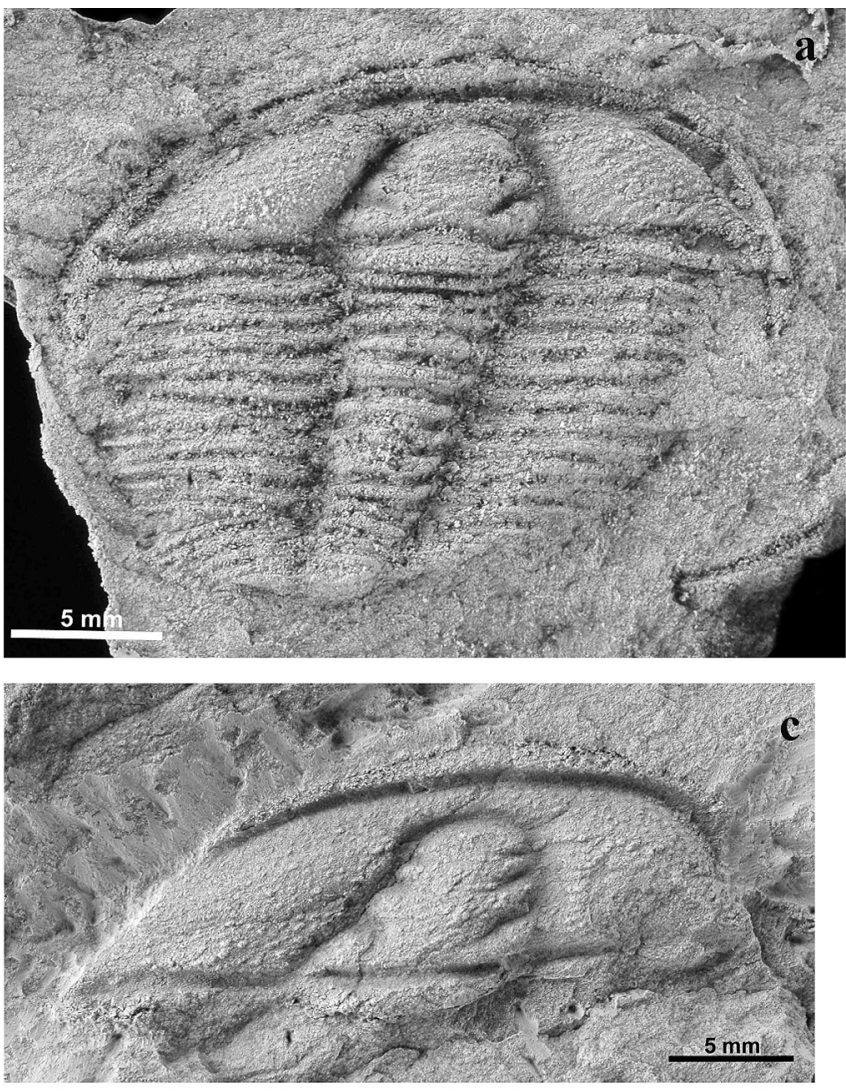
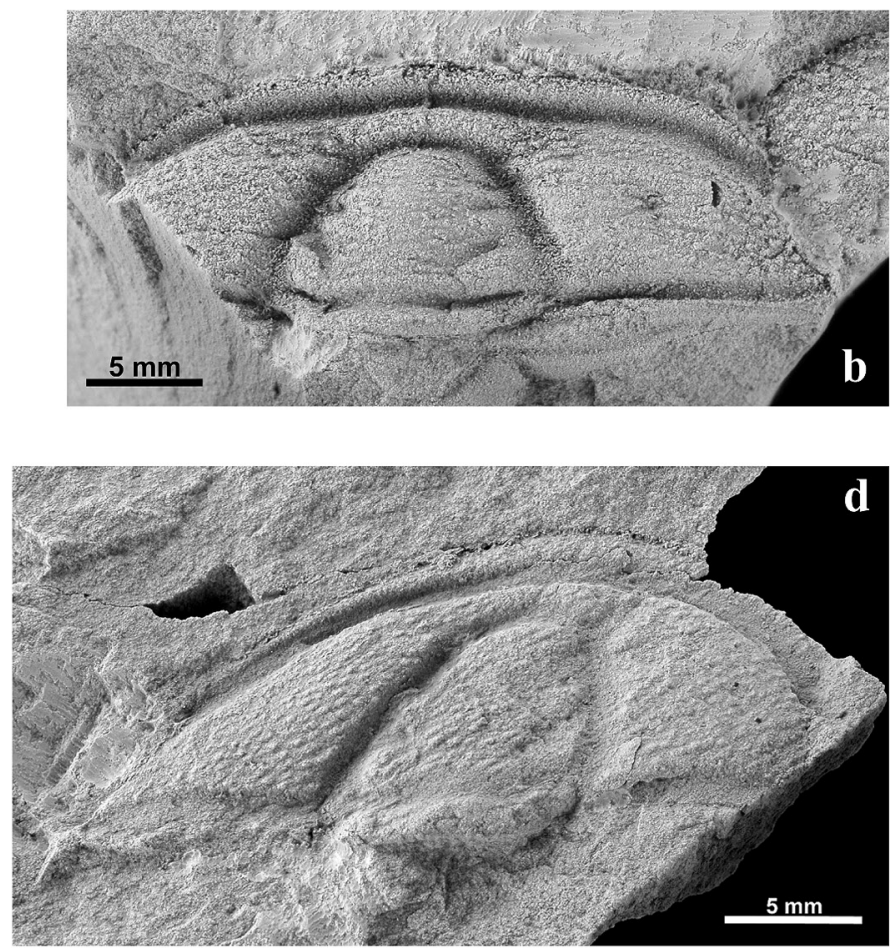
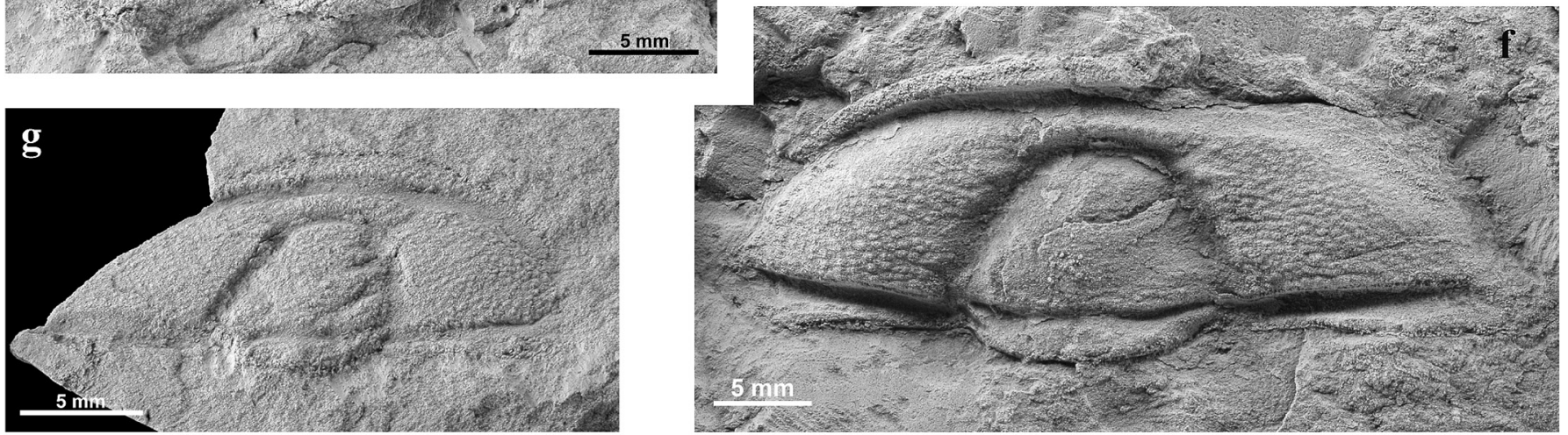

Figura 6. Parabailiella schmidti Sdzuy, 1957. a, molde interno de un ejemplar casi completo, MPZ2007/1293, RV1/13.1. b, molde interno de un cranidio, MPZ2007/1236, RV1/13.1. c, molde interno de un cranidio, MPZ2007/1234, RV1/13.1. d, molde interno de un cranidio, MPZ2007/1233, RV1/13.1. e, molde interno de un cranidio, MPZ2007/1237, RV1/13.1. f, molde interno de un cranidio, MPZ2007/1222, RV1/13.1.

Parabailiella schmidti Sdzuy, 1957. a, internal mould of an almost complete specimen, MPZ2007/1293, RV1/13.1. b, internal mould of a cranidium, MPZ2007/1236, RV1/13.1. c, internal mould of a cranidium, MPZ2007/1234, RV1/13.1.d, internal mould of a cranidium, MPZ2007/1233, RV1/13.1. e, internal mould of a cranidium, MPZ2007/1237.f, internal mould of a cranidium, MPZ2007/1222, RV1/13.1. 
coryphe, por debajo de la zona de Eccaparadoxides pusillus; este nivel presenta una edad que sería equivalente a un Leoniense medio, por lo que su edad sería ligeramente más antigua que el material procedente de España y Alemania.

En conjunto la especie tiene una distribución que abarca desde el Leoniense medio hasta los niveles basales del Caesaraugustiense medio, esto es, equivalente a parte del
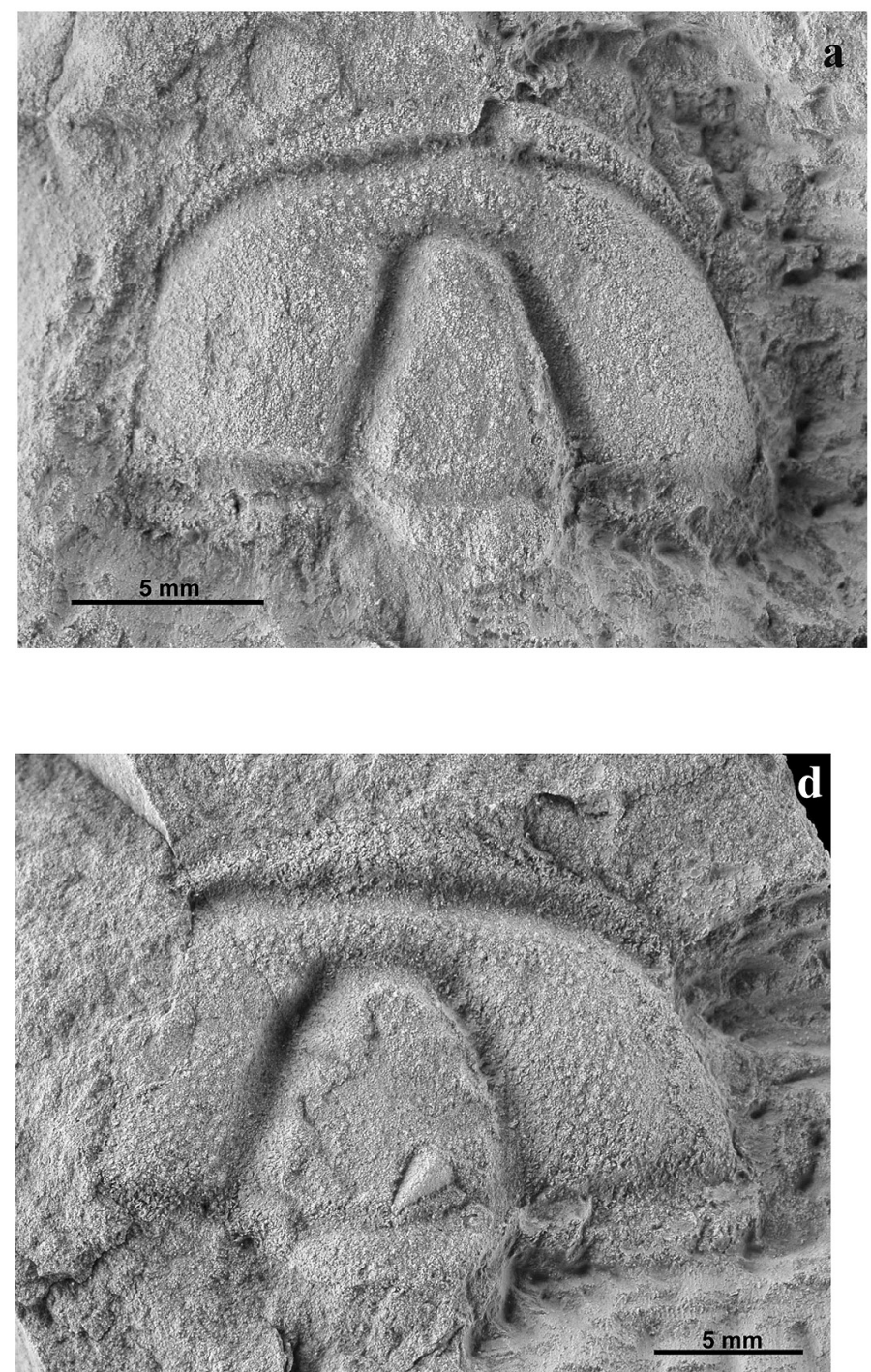

Piso 5 del Cámbrico y, posiblemente, la base del Drumiense.

\section{Parabailiella sebarensis Sdzuy, 1968}

Figura 7

* 1968. Conocoryphe (Parabailiella) sebarensis n. sp.- Sdzuy, 129-130, lám. 2 fig. 1, lám. 10 figs. 20-23.
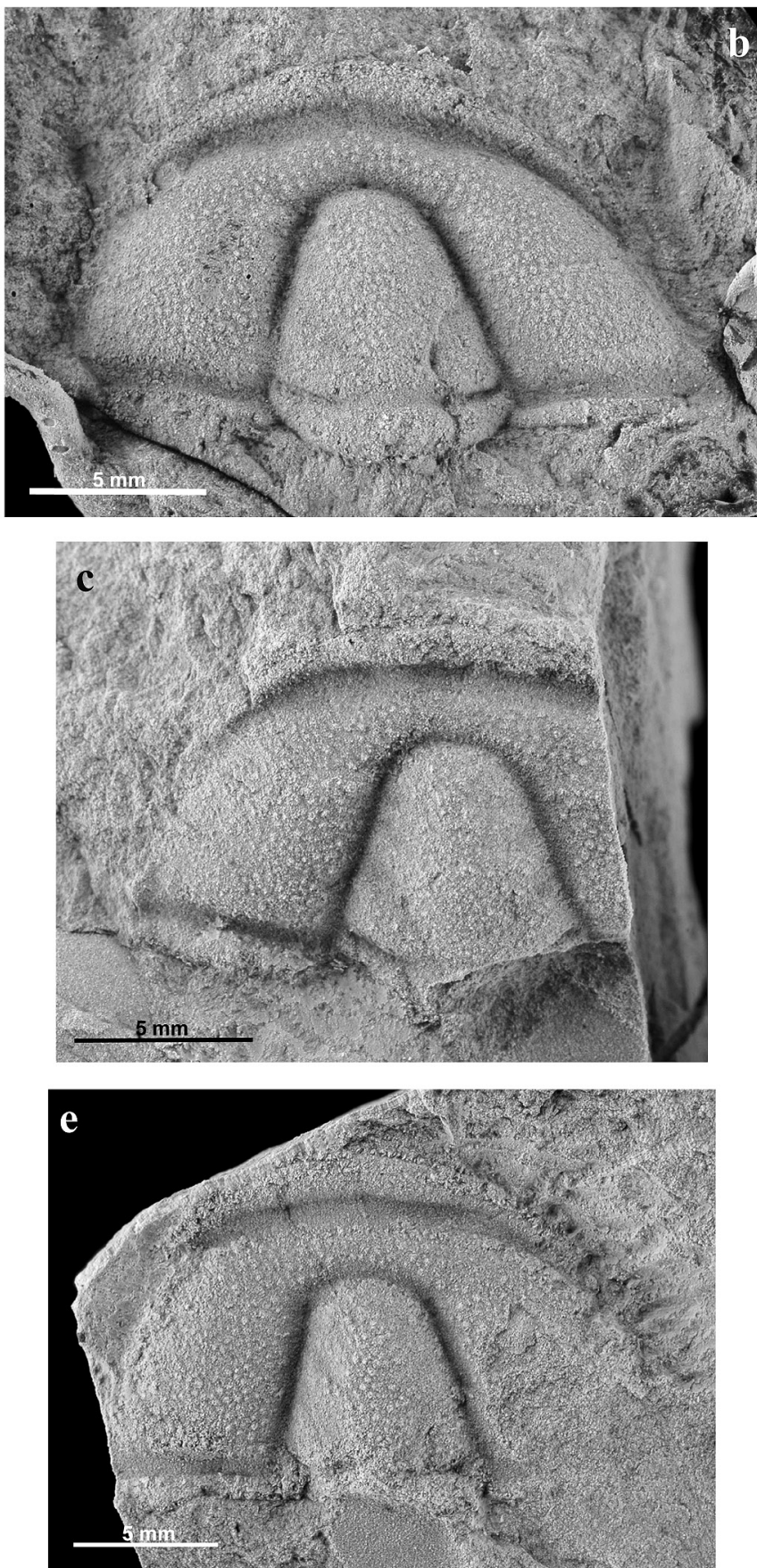

Figura 7. Parabailiella sebarensis Sdzuy, 1968. a, molde interno de un cranidio, MPZ2007/1353, J1/155. b, réplica de látex de un cranidio, MPZ2007/1352, J1/155. c, molde interno de un fragmento de cranidio, MPZ2007/1351. d, molde interno de un cranidio, MPZ2007/1343, J1/147F. e, molde interno de un cranidio, MPZ2007/1348, J1/147F.

Parabailiella sebarensis Sdzuy, 1968. a, internal mould of a cranidium, MPZ2007/1353, J1/155. b, latex cast of a cranidium, MPZ2007/1352, J1/155. c, internal mould of a cranidium fragment, MPZ2007/1351. d, internal mould of a cranidium, MPZ2007/1343, J1/147F. e, internal mould of a cranidium, MPZ2007/1348, J1/147F. 

1968.- Liñán Guijarro, 191-192, lám. XII fig. 1.

Material: Unos 30 cranidios conservados como moldes internos y externos en margas dolomíticas de color variado: verdes, rojizas y ocres claros, en lutitas ocres, rojas y verdes con algunos nódulos carbonatados dispersos. Los ejemplares estudiados tienen número de registro MPZ 2007/1335 al 2007/1364.

Descripción: Cranidio semicircular con sutura marginal que corta al surco posterior. El borde anterior es arqueado e inhomogéneo siendo más ancho en el centro y disminuyendo hacia los extremos, presenta ornamentación consistente en tubérculos dispuestos en dos o tres filas en la parte central que disminuyen hacia los laterales hasta desaparecer. El surco anterior es más ancho que profundo y no presenta ornamentación. El surco preglabelar es ancho y tampoco presenta ornamentación. El área preglabelar es abombada, no superando a la glabela ni al borde, está recorrida por más de dos filas de tubérculos finos y desordenados que se extienden por las fixígenas. La glabela es cónica con el ápice redondeado, está recorrida por tres pares de surcos no transglabelares que se dirigen hacia atrás y muy débilmente marcados, presenta ornamentación consistente en tubérculos desordenados. Anillo occipital en forma de semicírculo invertido, recorrido con la misma ornamentación que el resto del cranidio. Fixígenas abombadas sin superar en altura a la glabela, presentan la misma ornamentación en tubérculos finos que el resto del cranidio, junto al surco lateral de la glabela a una altura ligeramente inferior al ápice de la glabela, presenta un tubérculo más engrosado y en algunos ejemplares se observa el inicio de una arista ocular. El surco posterior es recto, ancho y liso.

Observaciones: Dentro de esta especie se han clasificado los ejemplares que presentaban aristas oculares rudimentarias, áreas preglabelares más anchas con tres o cuatro filas de tubérculos en su región axial y no tienen surcos preglabelares divergentes marcados que delimiten el área preglabelar del resto de la fixígena, lo que la diferencia de P. schmidti. Como ya se ha comentado en P. matutina, tanto en las Cadenas Ibéricas como en la Sierra de Córdoba estas dos especies se han encontrado de manera habitual juntas en los mismos niveles, aunque, de acuerdo con los datos de Sdzuy (1968) la aparición de la especie $P$. matutina fue previa a P. sebarensis.

Distribución estratigráfica: En las Cadenas Ibérica se ha encontrado en los niveles 1 y 2 de la Sección Mesones 3, nivel 5 de la Sección Borobia 3, niveles 6 y 7 de la Sección Borobia 2, y niveles $147 \mathrm{~F}$ al 160 de la Sección Jarque 1. Zonas de Eccaparadoxides asturianus y base de Badulesia tenera.

La distribución de esta especie en la Cordillera Cantábrica y en la Sierra de Córdoba se inicia en la zona de Eccaparadoxides asturianus y llega a la base de Badulesia tenera (Sdzuy, 1968; Liñán Guijarro, 1978; Perejón et al., 1996), datos que se han confirmado en este trabajo para las Cadenas Ibéricas. En conjunto tendría una distribución Leoniense superior hasta la base del Caesaraugustiense, esto es parte del Piso 5 del Cámbrico.

\section{AGRADECIMIENTOS}

Los autores quieren agradecer a las revisoras del trabajo, doctoras Isabel Rábano y $\mathrm{M}^{\mathrm{a}}$ Eugenia Dies, sus acertados comentarios que han ayudado a mejorarlo. Este trabajo es una contribución al proyecto Consolider CGL2006-12975/BTE ("MURERO) del Ministerio de Educación y Ciencia de España y FEDER, al Proyecto PM067/2006 del Gobierno de Aragón, al Programa Europa DGA (CNAI+D)-CAI y al Grupo Consolidado E-17 "Patrimonio y Museo Paleontológico del Gobierno de Aragón". Se agradece a la Sra. Isabel Pérez Urresti (técnico superior de proyectos financiados por el MEC, Fondo Social Europeo y UZ) por la realización de las fotografías. Esta es una contribución a la ISCS de la Unión Internacional de Ciencias Geológicas.

\section{REFERENCIAS}

Álvaro Blasco, J. J. 1994. El Cámbrico Inferior terminal y Medio de las Cadenas Ibéricas. Bioestratigrafía y Paleogeografía. Tesis doctoral, Universidad de Zaragoza, 250 pp. (inédita).

Álvaro, J. J. 2007. New ellipsocephalid trilobites from the lower Cambrian member of the Láncara Formation, Cantabrian Mountains, northern Spain. Memoir of the Association of Australasian Palaeontologists, 34, 343-355.

Álvaro, J. J. \& Vizcaïno, D. 2000. Nouvel assemblage de trilobites dans le Cambrien moyen de la nappe de Pardailhan (Montagne Noire, France): implications biostratigraphiques dans la région méditerranéenne. Eclogae geologicae Helvetiae, 93, 277-289.

Álvaro, J. J. \& Vizcaïno, D. 2003. The conocoryphid biofacies: a benthic assemblage of normal-eyed and blind trilobites. Special Papers in Palaeontology, 70, 127-140.

Álvaro, J. J., Vizcaïno, D. \& Vennin, E. 1999. Trilobite diversity patterns in the Middle Cambrian of southwestern Europe: a comparative study. Palaeogeography, Palaeoclimatology, Palaeoecology, 151, 241-254.

Angelin, N. P. 1854. Palceontologica Scandinavica. Pars II; Crustacea formationis transitionis. Academie Regiae Scientarium Suecanae, Fasc. 2, I-IX, 21-92, Holmiæ (Stockolm).

Babcock, L. E., Robison, R. A., Rees, M. N., Peng, S. \& Saltzman, M. R. 2007. The Global boundary Stratotype Section and Point (GSSP) of the Drumian stage (Cambrian) in the Drum Mountains, Utah, USA. Episodes, 30, 84-94.

Chirivella Martorell, J. B. 2008. Sistemática de los Trilobites, bioestratigrafía y paleoecología del límite LeonienseCaesaraugustiense (Cámbrico medio) en las Cadenas Ibéricas (NE de España). Tesis Doctoral, Universitat de València, $226 \mathrm{pp}$. (inédita).

Chirivella Martorell, J. B., Gozalo, R. \& Liñán, E. 2003. Estudio taxonómico y biométrico de Badulesia tenera (Hartt in Dawson, 1868), Trilobita. Caesaraugustiense (Cámbrico Medio) de las Cadenas Ibéricas (NE de España). Revista Española de Paleontología, 18, 83-101.

Cotton, T. J. 2001. The phylogeny and systematics of blind Cambrian ptychoparioid Trilobites. Palaeontology, 44, 167-207. 
Courjault-Radé, P., Debrenne, F. \& Gandin, A. 1992. Paleogeographic and geodynamic evolution of the Gondwana continental margins during the Cambrian. Terra Nova, 4, 657-667.

Courtessole, R. 1973. Le Cambrien Moyen de la Montagne Noire. Biostratigraphie. Imprimerie d'Oc, Toulousse, 248 pp.

Dean, W. T., Monod, O. \& Günay, Y. 1986. Lower Palaeozoic stratigraphy in the Southern and Central Amanos Mountains, south central Turkey. Geological Magazine, 123, 215-226.

Esteve, J., Gozalo, R., Liñán, E. \& Chirivella Martorell, J. B. 2008. Pardailhania Thoral, 1947 (Solenopleuropsinae, Trilobita) from Caesaraugustan (Middle Cambrian) of the Cadenas Ibéricas (NE Spain). Cuadernos del Museo Geominero, 9. Instituto Geológico y Minero de España, Madrid, 99-103.

Gámez, J. A., Fernández-Nieto, C., Gozalo, R., Liñán, E., Mandado, J. \& Palacios, T. 1991. Bioestratigrafía y evolución ambiental del Cámbrico de Borobia (Provincia de Soria. Cadena Ibérica Oriental). Cuadernos do Laboratorio Xeolóxico de Laxe, 16, 251-271.

Geyer, G. \& Landing, E. 2004. A unified Lower-Middle Cambrian chronostratigraphy for West Gondwana. Acta Geologica Polonica, 54, 179-218.

Geyer, G. \& Landing, E. 2006. Edicaran-Cambrian depositional environments and stratigraphy of the western Atlas regions. Beringeria, Special Issue, 6, 47-112.

Geyer, G., Landing, E. \& Heldmaier, W. 1995. Faunas and depositional environments of the Cambrian of the Moroccan Atlas regions. Beringeria, Special Issue, 2, 47-119.

Gozalo, R. \& Liñán, E. 1988. Los materiales hercínicos de la Cordillera Ibérica en el contexto del Macizo Ibérico. Estudios geológicos, 44, 399-404.

Gozalo, R., Liñán, E. \& Álvaro, J. 1993. Bioestratigrafía del Cámbrico Medio de Villafeliche (Prov. Zaragoza, España). Revista Española de Paleontología, no extr., 49-57.

Gozalo, R., Liñán, E. \& Sdzuy, K. 1996. September 20. The Lower and Middle Cambrian of Murero and Villafeliche (West Asturan-Leonese Zone. Western Iberian Chain). In: II Field Conference of the Cambrian Stage Subdivision Working Groups. International Subcommission on Cambrian Stratigraphy. Spain, 13-21 September 1996 (Eds. E. Liñán, J. A. Gámez Vintaned \& R. Gozalo). Field Trip Guide and Abstracts: Universidad de Zaragoza, 73-80.

Gozalo, R., Mayoral, E., Gámez Vintaned, J. A., Dies, M. E. \& Muñiz, F. 2003. A new occurrence of the genus Tonkinella in northern Spain and the Middle Cambrian intercontinental correlation. Geologica Acta, 1, 121-126.

Gozalo, R., Liñán, E., Dies, M. E., Gámez Vintaned, J. A. \& Mayoral, E. 2007. The Lower-Middle Cambrian boundary in the Mediterranean subprovince. In: The evolution of the Rheic Ocean: From Avalonian-Cadomian active margin to Alleghenian-Variscan collision (eds. U. Linnemann, R. D. Nance, P. Kraft \& G. Zulauf). Geological Society of America Special Paper, 423, 359-373.

Gozalo, R., Liñán, E., Gámez Vintaned, J. A., Dies Álvarez, M. E., Chirivella Martorell, J. B., Zamora, S., Esteve, J.
\& Mayoral, E. 2008. The Cambrian of the Cadenas Ibéricas (NE Spain) and its trilobites. Cuadernos del Museo Geominero, 9. Instituto Geológico y Minero de España, Madrid, 137-151.

Gozalo, R., Chirivella, J. B., Esteve, J. \& Liñán, E. 2009. Propuesta de correlación de la base del Drumiense (Serie 3 del Cámbrico) con la base del Caesaraugustiense medio (Cámbrico medio) en las Cadenas Ibéricas (NE de España). XXV Jornadas de la Sociedad Española de Paleontología, Libro de Resúmenes, 193-196.

Hawle, I. \& Corda, A. J. C. 1847. Prodom einer Monographie der böhmischen Trilobiten. Abhandlungen der Königliche böhmischen Gesellschaft der Wissenschaften, 5, 121-292.

Jell, P. A. \& Hughes, N. C. 1997. Himalayan Cambrian Trilobites. Special Papers in Palaeontology, 58, 1-113.

Kim, D. H., Westrop, S. R. \& Landing, E. 2002. Middle Cambrian (Acadian series) conocoryphid and paradoxidid trilobites from the Upper Chamberlain's Brook Formation, Newfounland and New Brunswick. Journal of Paleontology, 76, 822-842.

Korobov, M. N. 1966. New trilobites of the family Conocoryphidae from the Cambrian of Siberian Platform and Tuva. Palaeontologischeskiy Zhurnal, 1966, 92-97 (en ruso).

Korobov, M. N. 1973. Trilobites of the Conocoryphidae family and their importance for the stratigraphy of Cambrian deposits. Transactions of the Academy of Science of the USSR, geological Institute, 211, 1-176 (en ruso).

Liñán Guijarro, E. 1978. Bioestratigrafía de la Sierra de Córdoba. Tesis doctorales de la Universidad de Granada, 191, 1-212.

Liñán, E. \& Gozalo, R. 1986. Trilobites del Cámbrico Inferior y Medio de Murero (Cordillera Ibérica). Memorias del Museo Paleontológico de la Universidad de Zaragoza, 2, 1-104.

Liñán, E. \& Sdzuy, K. 2001. Trilobites del Cámbrico Medio de Cuevas del Sil (Noroeste de España). In: La era Paleozoica. El desarrollo de la vida marina. Homenaje al Profesor Jaime Truyols (eds. J. A. Gámez Vintaned \& E. Liñán). Institución "Fernando el Católico", Zaragoza, 251-265.

Liñán, E., Gozalo, R., Gámez, J. A. \& Álvaro, J. J. 1992. Las formaciones del Grupo Mesones (Cámbrico InferiorMedio) en las Cadenas Ibéricas. III Congreso Geológico de España y VIII Congreso Latinoamericano de Geología, Salamanca, Actas, 1, 517-523.

Liñán, E., Álvaro, J. J., Gozalo, R., Gámez-Vintaned, J. A. \& Palacios, T. 1995. El Cámbrico Medio de la Sierra de Córdoba (Ossa-Morena, S de España): trilobites y paleoicnología. Implicaciones bioestratigráficas y paleoambientales. Revista Española de Paleontología, 10, 219-238.

Liñán, E., Sdzuy, K., Álvaro, J. J., Gámez Vintaned, J. A. \& Gozalo, R. 1996. September 19. The Jarque section: Lower and Middle Cambrian (West Asturian-Leonese Zone. Eastern Iberian Chain). In: II Field Conference of the Cambrian Stage Subdivision Working Groups. International Subcommision on Cambrian Stratigraphy. Spain. 13-21 September 1996. Field Trip Guide and Abstracts 
(Eds. E. Liñán, J. A. Gámez Vintaned \& R. Gozalo). Universidad de Zaragoza, 57-72.

Liñán, E., Gonçalves, F., Gámez Vintaned, J. A. \& Gozalo, R. 1997. Evolución paleogeográfica del Cámbrico de la Zona de Ossa-Morena basada en el registro fósil. In: Estudo sobre a Geologia da Zona de Ossa-Morena (Maciço Ibérico). Homenagem ao Prof. Francisco Gonçalves (eds. A. A. Araújo \& M. F. Pereira). Universidade de Évora, 1-26.

Liñán, E., Gozalo, R., Palacios, T., Gámez Vinaned, J. A., Ugidos, J. M. \& Mayoral, E. 2002. Cambrian. In: The Geology of Spain (eds. W. Gibbons \& T. Moreno). The Geological Society, London, 17-29.

Liñán, E., Perejón, A., Gozalo, R., Moreno-Eiris, E. \& Oliveira, J. T. de 2004. The Cambrian System in Iberia. Cuadernos del Museo Geominero, 3. Instituto Geológico y Minero de España, Madrid, 63 pp.

Liñán, E., Gozalo, R., Dies Álvarez, M. E., Gámez Vintaned, J. A., Mayoral, E., Chirivella Martorell, J. B., Esteve, J., Zamora, S., Zhuravlev, A. Yu. \& Andrés, J. A. 2008. Fourth International Trilobite Conference Trilo 08 Toledo, Spain, 2008. Post-Conference Field Trip. Lower and Middle Cambrian trilobites of selected localities in Cadenas Ibéricas (NE Spain). Universidad de Zaragoza, 52 pp.

Mansuy, H., 1916. Faunes cambriennes de l'extrèmeOrient méridional. Mémoires du Service Géologique de l'Indochine, 5, 1-48.

Matthew, G. F. 1885. Illustrations of the fauna of the St. John Group, continued: On the Conocoryphea, with further remarks on Paradoxides. Transactions of the Royal Society of Canada, section 4, 2, (1884), 9-124

Matthew, G. F. 1888. Illustrations of the Fauna of the St. John Group. No. IV.- Part I. Description of a new species of Paradoxides (Paradoxides regina). Part II. The smaller trilobites with eyes (Ptychoparidæ and Ellipsocephalidæ). Transactions of the Royal Society of Canada, section 4, 5, (1887), 115-166.

Matthew, G. F. 1899. Studies on Cambrian faunas, No. 4.Fragments of the Cambrian Faunas of Newfoundland. Proceedings and Transactions of the Royal Society of Canada, 2nd Serie, 5, 67-95.

Özdikmen, H. 2009. Nomenclatural changes for twenty trilobites genera. Munis Entomology \& Zoology, 4, 155-171.

Perejón, A., Moreno-Eiris, E., Liñán, E. \& Sequeiros, L. 1996. September 16. The Cambrian of the Sierra de Córdoba. (Ossa-Morena Zone. Sierra Morena). In: II Field Conference of the Cambrian Stage Subdivision Working Groups. International Subcommision on Cambrian Stratigraphy. Spain. 13-21 September 1996. Field Trip Guide and Abstracts (Eds. E. Liñán, J. A. Gámez Vintaned \& R. Gozalo). Universidad de Zaragoza, 45-56.

Resser, C. E. 1936. Second Contribution to Nomenclature of Cambrian Trilobites. Smithsonian Miscellaneous Collections, 95, 1-29.
Richter, R. 1932. Crustacea (Paläontologie). In: Handwörterbuch der Naturwissenschaften, 2nd ed. (Eds. R. Dittler, G. Joos, E. Korschelt, G. Linek, F. Oltmanns \& K. Schaum). Gustav Fisher, Jena, 840-864.

Salter, J. W. 1864. A Monograph of the British Trilobites from the Cambrian, Silurian and Devonian formations. Part 1. Monographs of the Palaeontographical Society, 16 (67), (1862), 1-80.

Schmidt, E. W. 1942. Die mittelkambrische Fauna von Doberlug. Jahrbuch des Reichsamts für Bodenforschung, 62, 344-402.

Sdzuy, K. 1957. Revision der mittelkambrischen Trilobiten von Doberlug. Senckenbergiana lethaea, 38, 7-28.

Sdzuy, K. 1961. Das Kambrium Spaniens. Teil II: Trilobiten. Akademie der Wissenschaften und der Literatur Abhandlungen der Mathematisch-Naturwissenschaftililichen Klasse, 1961 (7-8), 217-408.

Sdzuy, K. 1968. Trilobites del Cámbrico Medio de Asturias. Trabajos de Geología, Universidad de Oviedo, 1, (1967), 77-133.

Sdzuy, K. 1972. Das Kambrium der acadobaltischen Faunenprovinz. Zentralblatt für Geologie und Paläontologie, Teil II, 1972, 1-91.

Sdzuy, K. \& Liñán, E. 1996. Cornucoryphe schirmi n. gen. n. sp., an unusual conocoryphid trilobite from the Middle Cambrian of Spain. Paläontologische Zeitschrift, 70, 433-438.

Sdzuy, K., Liñán, E. \& Gozalo, R. 1999. The Leonian Stage (early Middle Cambrian): a unit for Cambrian correlation in the Mediterranean subprovince. Geological Magazine, 136, 39-48.

Shergold, J. H. \& Sdzuy, K. 1984. Cambrian and Early Tremadocian trilobites from Sultan Dag, Central Turkey. Senckenbergiana lethaea, 65, 51-135.

Swinnerton, H. H. 1915. Suggestions for a revised classification of trilobites. Geological Magazine, new series, 6 , 487-496, 538-545.

Thoral, M. 1946. Conocoryphidae Languedociens. Annales de l'Université de Lyon, série $3^{\text {eme }}$, section $C$, Sciences naturelles, 4, 1-74.

Valenzuela, J. I., Gámez, J. A, Liñán, E. \& Sdzuy, K. 1990. Estratigrafía de la región de Brea. Cadena Ibérica Oriental. Boletín de la Real Sociedad Española de Historia Natural (Sección Geológica), 85, 45-54.

Valíček, J. \& Szabad, M. 2002. Revision early Middle Cambrian trilobite fauna from Bohemia. Palaeontologia Bohemiae, 8, 75-98.

Westergård, A. H. 1950. Non-Agnostidean Trilobites of the Middle Cambrian of Sweden. II. Sveriges Geologiska Undersökning, ser. C, 511, 1-57.

Manuscrito recibido: 15 de Noviembre, 2009 Manuscrito aceptado: 30 de Diciembre, 2009 